\title{
Parallel spike trains analysis using positive definite kernels
}

\author{
Taro Tezuka \\ From The Twenty Third Annual Computational Neuroscience Meeting: CNS*2014 \\ Québec City, Canada. 26-31 July 2014
}

Since spikes are considered to be the fundamental element in neural systems, there has been a significant amount of work that analyzes the sequence of spike timings, or spike trains $[1,2]$. One important research question is to know how information is encoded in a spike train. There is an approach to construct a feature space that captures characteristics of spike trains, as is usually done in the field of pattern recognition. Another approach, which is increasingly becoming popular in recent years, is to find an appropriate similarity measure between spike trains $[3,4]$. This corresponds to constructing a model of information decoding done by the postsynaptic neurons.

One promising way to define such similarity measures is to use positive definite kernels [5-7]. Since positive definite kernels are generalizations of inner products, they enable application of various linear methods used in machine learning (including regression, classification, and dimension reduction) to spike trains. Positive definite kernels are extensively used in kernel methods.

While positive definite kernels on spike trains obtained from a single neuron have already been proposed [5-7], the extension of this to parallel spike trains obtained from multiple neurons has not been explored yet. Therefore, we defined such a kernel by extending the memoryless cross intensity kernel (mCI kernel) proposed by Paiva et al. [5]. To make the extension as natural as possible, we used a linear combination of cross-neuron interactions. We name the new kernel the linear combination of interactions kernel (LCIK) [9]. The parameters of the kernel can be set to make it positive definite.

We applied this kernel to publicly available in vivo recordings taken from the primary visual field in macaque monkey brains [8]. The LCIK performed better than other possible kernels defined on a set of spike trains. When the

Correspondence: tezuka@slis.tsukuba.ac.jp

Faculty of Library, Information, and Media Science, University of Tsukuba, Tsukuba, 305-0821, Japan parameters of the kernel were estimated using spike trains obtained from real neurons, we obtained biologically plausible values. For example, the estimated time constant was near the value commonly used in neural modeling. This indicates the possibility of using such kernels to look for an appropriate model of neurons based on observed spike recordings.

We also simulated a simple neural network and generated spike trains to see the effect on the estimated values of the kernel parameters by changing the synaptic connection parameters. The result indicated that the kernel indirectly represents some of the internal parameters of the neural networks.

Acknowledgement

This work supported in part by JSPS KAKENHI Grant Numbers 21700121,

Published: 21 July 2014

\section{References}

Gerstner W, Kistler W: Spiking Neuron Models. Cambridge: Cambridge University Press; 2002.

2. Rieke F, Warland D, de Ruyter van Steveninck R, Bialek W: Spikes: Exploring the Neural Code. Cambridge, MA: MIT Press; 1997.

. Victor JD: Spike train metrics. Current Opinion in Neurobiology 2005, 15:585-592.

4. Houghton C, Sen K: A new multi-neuron spike-train metric. Neural Computation 2008, 20:1495-1511.

5. Paiva ARC, Park I, Principe JC: Inner products for representation and learning in the spike train domain. Statistical Signal Processing for Neuroscience and Neurotechnology Academic Press; 2010.

6. Park I, Seth S, Rao M, Principe JC: Strictly positive definite spike train kernels for point process divergences. Neural Computation 2012 24:2223-2250 spike train data. Advances in Neural Information Processing Systems 2010, 23:595-603,

8. Aronov D, Reich DS, Mechler F, Victor JD: Neural coding of spatial phase in V1 of the macaque monkey. J Neurophysiology 2003, 89:3304-3327.

9. Tezuka T: Spike train kernels for multiple neuron recordings. Proceedings of the 39th International Conference on Acoustics, Speech and Signal Processing 2014, (to appear).
7. Fisher NK, Banerjee A: A novel kernel for learning a neuron model from 

and take full advantage of:

- Convenient online submission

- Thorough peer review

- No space constraints or color figure charges

- Immediate publication on acceptance

- Inclusion in PubMed, CAS, Scopus and Google Scholar

- Research which is freely available for redistribution 